\title{
Editorial
}

\section{Wearable and RFID Antennas}

\author{
Duixian Liu, ${ }^{1}$ Atif Shamim, ${ }^{2}$ Tzyh-Ghuang Ma, ${ }^{3}$ and Zhinong Ying ${ }^{4}$ \\ ${ }^{1}$ IBM Watson Research Center, Yorktown Heights, NY 10598, USA \\ ${ }^{2}$ Division of Physical Sciences and Engineering, Electrical Engineering Department, King Abdullah University of Science and \\ Technology (KAUST), Thuwal 23955-6900, Saudi Arabia \\ ${ }^{3}$ Department of Electrical Engineering, National Taiwan University of Science and Technology, Taipei 10607, Taiwan \\ ${ }^{4}$ Network Research Laboratory, Research and Technology, CTO Office, Sony Ericsson Mobile Communication AB, \\ 22188 Lund, Sweden \\ Correspondence should be addressed to Duixian Liu; duixian@us.ibm.com
}

Received 1 August 2013; Accepted 1 August 2013

Copyright ( 2013 Duixian Liu et al. This is an open access article distributed under the Creative Commons Attribution License, which permits unrestricted use, distribution, and reproduction in any medium, provided the original work is properly cited.

The desire of accessing information everywhere and anytime has led to a surge in wireless communication devices. Numerous new applications are being explored, and, as a result, innovative devices are being envisioned. The applications span from wearable sensing and communication for body area networks to real time tracking and localization of personnel and objects. Primary requirements for these new devices are small form factor, light weight, low cost, conformal, and preferably environmentally friendly. To achieve lower cost, fabrication on low cost organic substrates such as polymers is being sought. Organic and polymer substrates are cheaper, lighter, and, in the case of organics, more environmentally friendly with the additional advantage of providing flexibility. The size of such devices is typically dictated by the size of the antennas, so miniaturization of antennas without losing the efficiency is an important issue. In addition to small form factors, such antennas are also expected to support multiband operations, provide ease of integration with driving circuits, and, most importantly, are of low cost. This special issue is focused on various aspects of antenna designs which are suitable for such futuristic applications.

G. Liu et al., show an interesting method of miniaturizing the traditional patch antenna through a special cross-shaped fractal metamaterial structure that is inserted between the patch and ground plane. With this method, they demonstrate a size reduction of more than $10 \%$ for the $900 \mathrm{MHz}$ band. The design is highly suitable for numerous UHF RFID based applications.
$\mathrm{H}$. Liu et al., present an interesting design concept to achieve miniaturization and capability of integrating multisystems of wireless communication terminals through a new ultrawideband planar inverted $\mathrm{F}$ antenna (PIFA) design. The PIFA employs a capacitive ground plane which is composed of a sheet of metal islands. By applying the capacitive compensation for input impedance of the PIFA in high-order modes frequency bands, ultra-wideband operation from $2.3 \mathrm{GHz}$ to 9.0 GHz is achieved with a small form factor.

G. Liu et al., demonstrate a novel high performance design for global positioning system (GPS) dual-band operation $(1.575 \mathrm{GHz}$ and $1.227 \mathrm{GHz}$ for L1 band and L2 band, resp.). The design employs two separate circularly polarized antennas with good isolation. To enhance the gain at low angle, a new structure of patch and two parasitic metal elements are introduced in this work. With the optimized design, good axial ratio and near hemispherical radiation pattern are obtained.

L. Zhao et al., investigate the effects of reentry plasma sheath on a GPS right-hand circularly polarized (RHCP) patch antenna polarization property during a typical reentry process. The very important result which they report is that the GPS RHCP patch antenna radiation pattern is distorted along with deteriorations in polarization during the entire reentry process and that appropriate measures must be taken to maintain the navigation communication.

H. Liu et al., study the characteristics of directivity enhancement using different metamaterial structures as antenna superstrates, such as electromagnetic bandgap (EBG) 
structures, frequency selective surface (FSS), and left-handed material (LHM). The study focuses on the analysis of reflection phase and magnitude of superstrates, and the essential reason for high-directivity antenna with different superstrates is revealed in terms of the Fabry-Perot resonant theory.

$\mathrm{X}$. Yang et al., provide a theoretical analysis to improve the body area line-of-sight probability density model. They prove that the traditional least-square statistical model is not necessarily the best choice when only limited samples can be collected. The proposed method helps to improve the probability density estimation in "body area network" radio propagation characterization.

These manuscripts present interesting design insights for miniaturized, multiband, and low cost antennas suitable for RFID, navigation, and wearable (body area network) applications. Emerging designs with enhanced performances, overcoming existing challenges, have been included in this special issue, which may inspire the reader and help advance the present research on the topic. We would like to thank all authors, reviewers, and the guest editor for making this special issue on RFID and wearable antennas possible.

Duixian Liu Atif Shamim

Tzyh-Ghuang Ma Zhinong Ying 

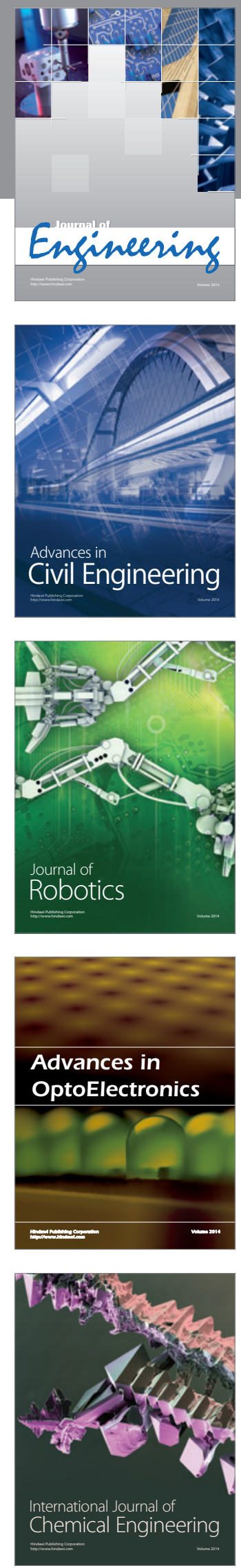

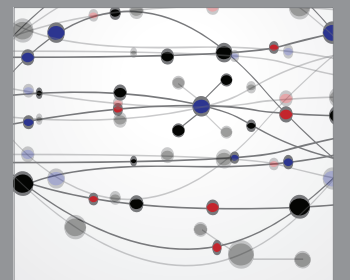

The Scientific World Journal
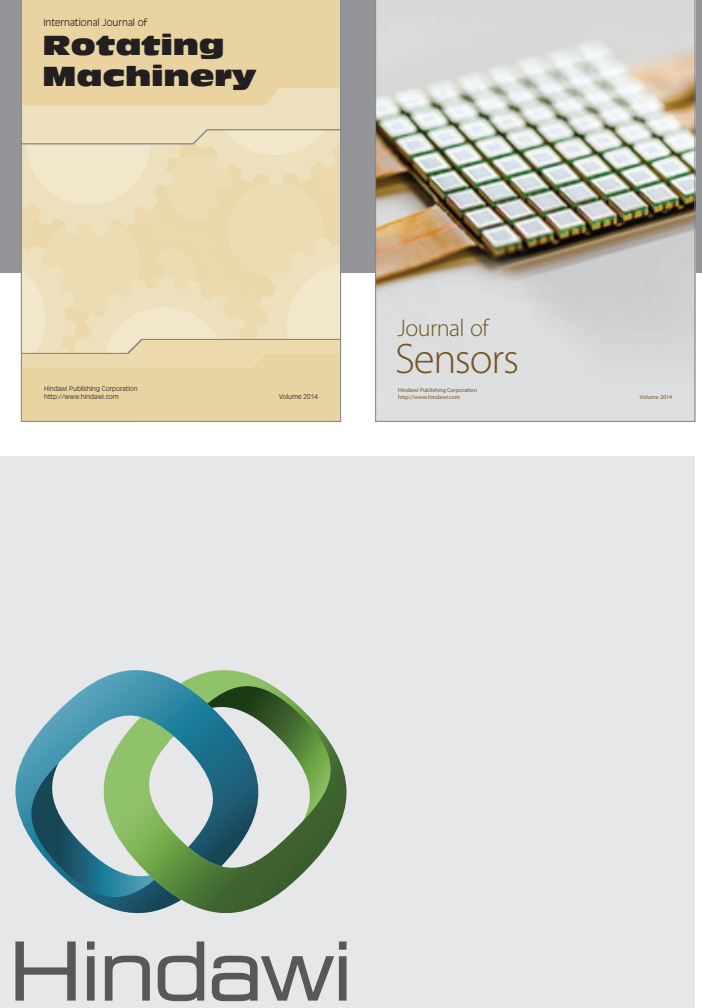

Submit your manuscripts at http://www.hindawi.com
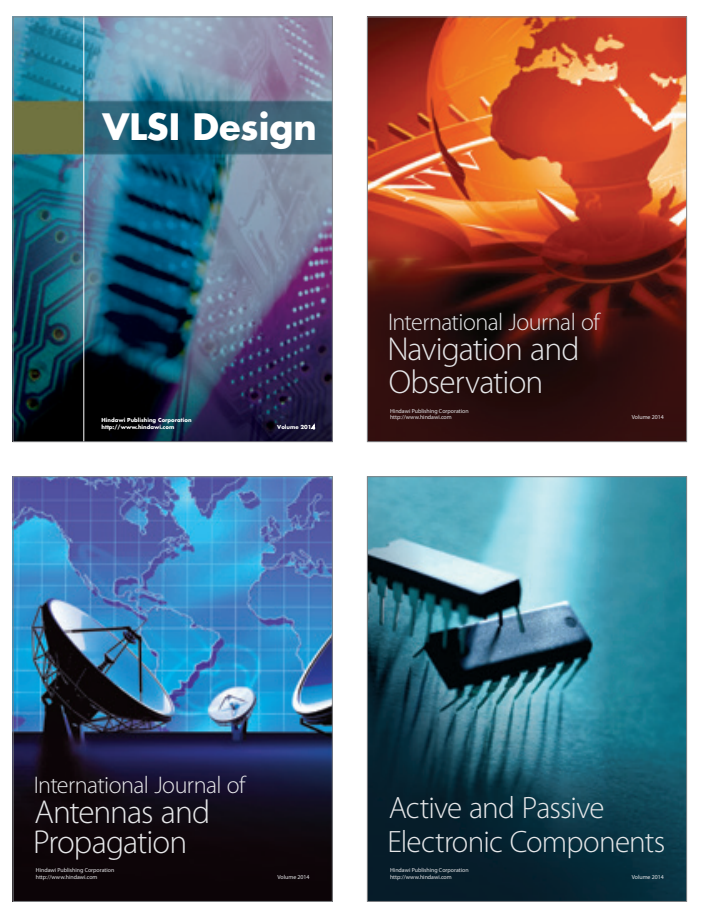
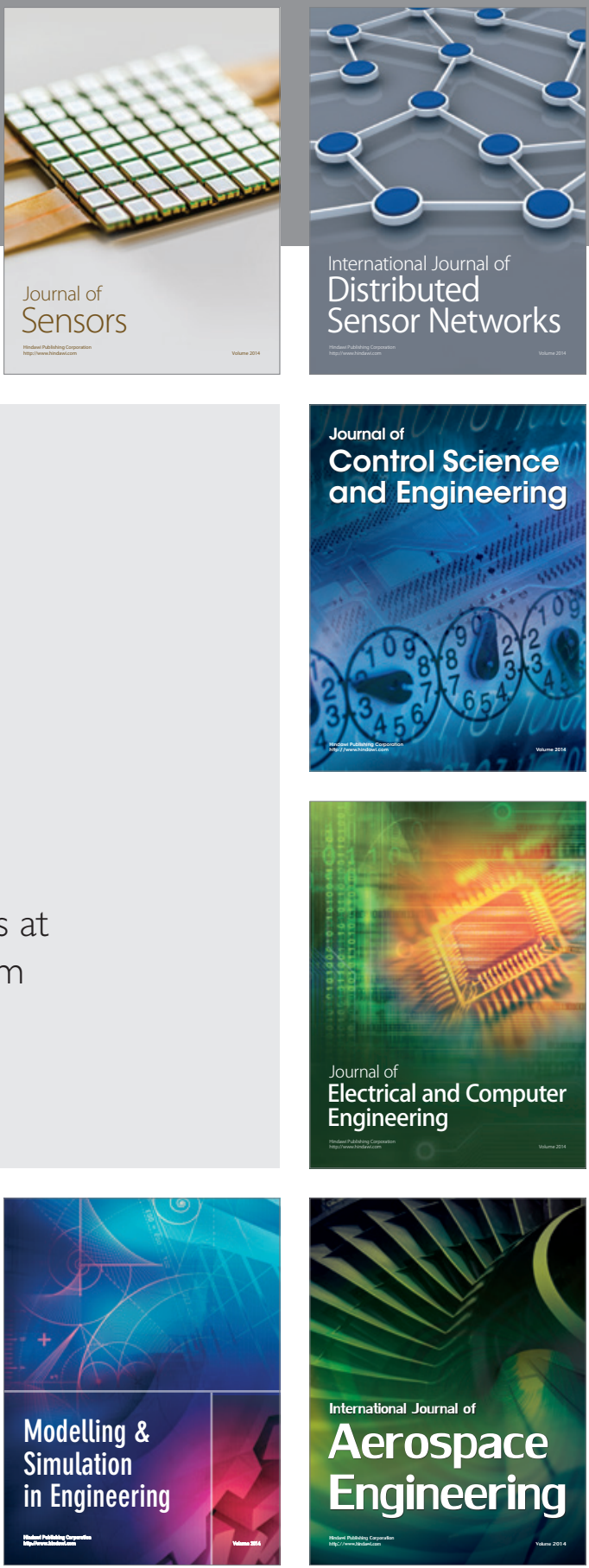

Journal of

Control Science

and Engineering
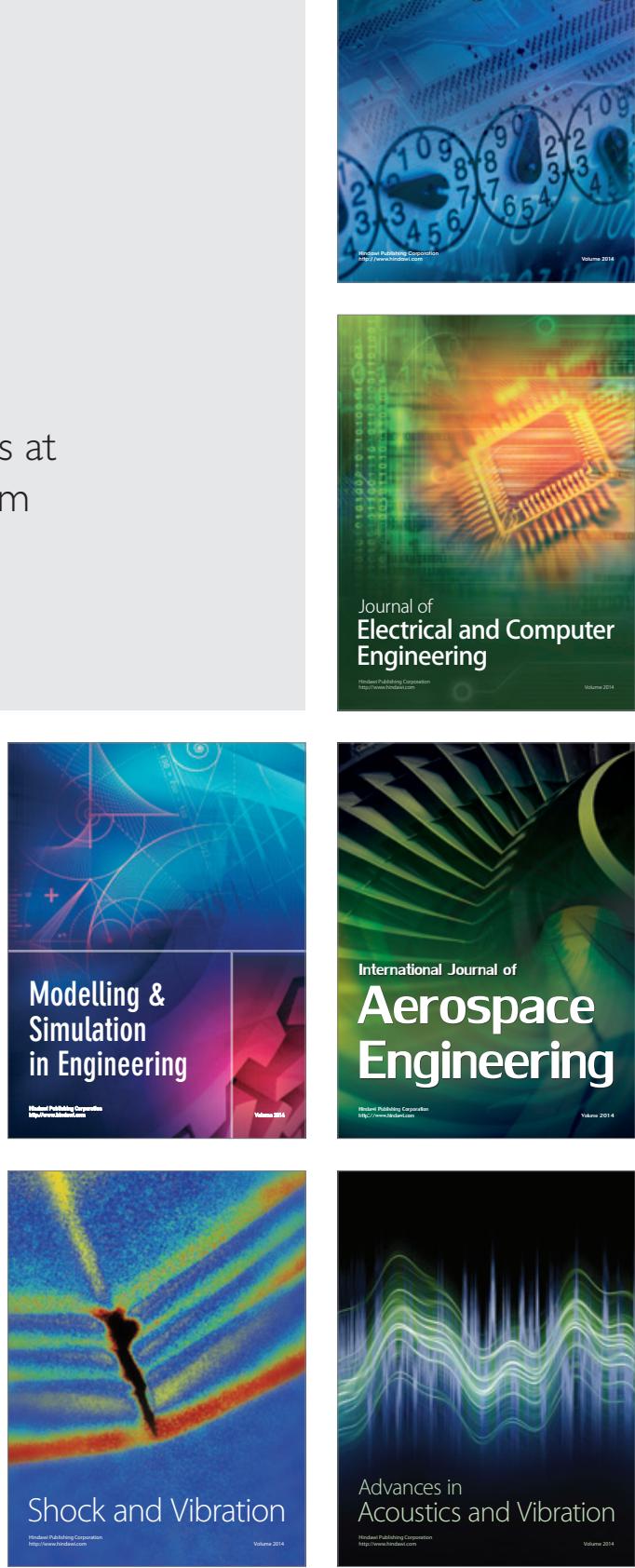